\title{
LA NUEVA SOLTERIA DE LOS CATALANES
}

RESUMEN. En algunos países de Europa, la tendencia al alargamiento del calendario de la nupcialidad había redundado en una elevación de la edad media al matrimonio y en un incremento de la soltería definitiva. En España, parece haber sucedido lo mismo. Esta inversión de la anterior tendencia de rejuvenecimiento de la nupcialidad se destaca como notoria en la década del 80 . Tanto en Cataluña como en la ciudad de Barcelona se observan las menores proporciones de solteros y solteras en casi todos los grupos de edad, a partir de los datos censales de 1981. Esto implicaría que es la última fecha y punto culminante de una tendencia. La década del 80 va a significar la alteración y el cambio en materia de nupcialidad. Esta notoria postergación del casamiento que han decidido los jóvenes catalanes no ha sido tan dilatada. Signos de recuperación se observan a partir del segundo quinquenio. Esto no implicaría volver a adoptar viejos comportamientos. Las nuevas generaciones son portadoras de cambios en las costumbres y es preciso analizarlos.

\section{INTRODUCCION}

La tendencia de este siglo a casarse cada vez más y más joven se ha invertido. En Cataluña, el Censo de 1981 registraba las menores proporciones de solteros y solteras del último período. A partir de esa fecha, los datos padronales sobre estado civil muestran comportamientos distintos. La nupcialidad de esta década ha cambiado también: los casamientos se hacen más raros y más tardíos, tanto para los hombres como para las mujeres. 
Una alteración de estas características es digna de estudio y consideración, pero lo es mucho más por el ritmo y la velocidad del cambio registrado que por el hecho en sí. Observando las tendencias europeas en materia de nupcialidad, podemos decir que los nuevos comportamientos fueron predecibles. Pero la rapidez con que las nuevas generaciones los han adoptado, sorprende.

Evidentemente, siempre se hablaba de un cierto retardo en las mag. nitudes y las tendencias de las variables demográficas españolas respecto a otros países europeos occidentales. Este espacio de tiempo, que había sido cifrado en veinte años primero, y luego sucesivamente reducido, tiende a desaparecer. Los comportamientos de las nuevas generaciones de españoles y españolas se asemejan cada vez más al resto de habitantes europeos, sin necesidad de que midamos las diferencias en términos de intervalos necesarios para alcanzar los niveles de los restantes países.

En este nuevo contexto, los habitantes de Cataluña presentan iguales tendencias que en el conjunto de España. En el pasado se podía hacer hincapié en las diferencias regionales más que ahora, en la medida en que las personas que habitaban en una comunidad podían haber nacido mayoritariamente en ella. A posteriori, debido a las importantes migraciones internas, la heterogeneidad por origen de los habitantes es una de las características de las sociedades de recepción - flujos positivos-... En esa medida, cuando se pudo distinguir el comportamiento frente a la nupcialidad de las personas residentes en Barcelona, por lugar de nacimiento, se constataron diferencias importantes. Tanto los hombres como las mujeres nacidos en Cataluña se quedaban solteros en mayor proporción que el resto de los habitantes de esta Comunidad Autónoma.

La posibilidad de contrastar y comparar las distintas subpoblaciones según el lugar de origen ha permitido insistir en esta peculiaridad. Por ello, puedo afirmar que el contexto urbano y metropolitano de la Ciudad Condal ha tenido una influencia negativa en la probabilidad de casarse de las personas nacidas en ella y en la Comunidad Autónoma a la que la ciudad pertenece, pero no se ha manifestado de igual manera en las que provenían de fuera (Sarrible, 1987).

El objetivo de este artículo es destacar los nuevos comportamientos adoptados en materia de nupcialidad, en la década de los ochenta, de los habitantes de esta Comunidad Autónoma, comparándolos con los ciudadanos de Barcelona. En múltiples trabajos, de diversas especialidades de las ciencias sociales, se ha comprobado que ciertas características o tendencias que se vislumbran y observan en los habitantes de la ciudad se extienden paulatinamente al resto de la población. La distinta composición socioprofesional de ésta - como de otras ciudades - bajo el supuesto de la imitación social permitiría formular la hipótesis de un comportamiento vanguardista de los habitantes urbanos. 
El estudio de la nupcialidad muchas veces se reduce al del sexo femenino como prolegómeno de los temas de fecundidad. En este caso he querido analizar la conducta frente al casamiento de ambos sexos. Primero, porque noto a la vez una cierta aproximación o similitud en los comportamientos por la edad -la pertenencia a las mismas o próximas generaciones- y una diferenciación cada vez más notable en términos de género. Todos los jóvenes avanzan y contribuyen a ahondar los cambios, pero las actitudes y conductas de los hombres son distintos a los de las mujeres ${ }^{1}$.

\section{LOS DATOS}

\section{Las fuentes}

Los censos y padrones realizados cada quinquenio permiten la utilización del método de Hajnal para calcular las proporciones de solteros y solteras y poder estudiar el estado civil de la población en cada una de esas fechas. Para Cataluña y Barcelona ya se puede disponer de los resultados del último Padrón de 1986 por edad, sexo y estado civil.

A partir del Movimiento Natural de la Población, del INE, se han podido calcular las tasas de nupcialidad de solteros y solteras, de segunda categoría, para el período 1980-1983. En el Movimiento Natural de la Población, publicado por el CIDC, se recogen los matrimonios de los años 1985 y 1986, para el conjunto de la Comunidad Autónoma. Queda, pues, un año sin poder realizar estimaciones ni calcular indicadores para la población catalana y un período abierto que se inicia en 1984 para la ciudad de Barcelona.

Resulta lamentable, sobre todo en un período de cambios y alteraciones, no poder contar con los datos del año 1984. Sin embargo, el salto entre los cuatro primeros años de la década y la mitad es cualitativamente tan destacado que me parece importante poder reflexionar sobre ello, incluso como contraste. Una cierta recuperación comienza a ser observada, a nivel de toda Cataluña, en la intensidad de la nupcialidad.

\section{Las proporciones de solteros y solteras}

Si se consideran tres fechas de estudio, coincidentes con los últimos padrones, la del medio, 1981, puede constituir el punto de referencia de lo su-

1 Tanto el período a estudio es breve como los cambios concentrados. No intento un análisis de la nupcialidad en el largo plazo, sino constatar las nuevas tendencias en este aspecto. Es por ello que este trabajo está limitado a un período de tiempo relativamente reducido. Contrastar las tendencias de la década anterior con la presente basta para visualizar las alteraciones producidas. La cantidad de datos publicados también es reducida, por lo que me cerniré a señalar las transformaciones acaecidas. 
cedido antes y después de ese momento. Fijar los cambios en la década del ochenta implica una línea divisoria, respecto a las tendencias en el pasado, que pueden alterarse o invertirse durante estos últimos años.

Los datos del Padrón y del Censo de 1981 reflejan tanto el punto máximo de una nupcialidad cada vez más intensa y más temprana como el último vestigio de este comportamiento. Sin embargo, al considerar las distintas unidades de estudio - Cataluña y Barcelona, provincia y ciudad-, podemos destacar ciertas diferencias para las generaciones que están próximas a salir de observación y tienen edades relativamente elevadas. Estos hombres y mujeres no se han visto afectados por el cambio y en algunos casos han continuado con la anterior tendencia a casarse más que otras generaciones más antiguas en anteriores años.

En el cuadro 1 constan las proporciones de solteros en Cataluña en 1975, 1981 y 1986. Resulta evidente el proceso de rejuvenecimiento. Casi uno de cada cuatro varones ya se había casado en el grupo 20-24, y menos de un tercio quedaba soltero en el grupo de edad siguiente, en 1981. Estas bajas proporciones contrastan con las cifras de 1986, superiores, en todos los casos, a las correspondientes a 1981 y aun a las estimadas para 1975. Esto implica que el proceso de rejuvenecimiento ha llegado a su fin y que en pocos años se ha retrocedido a niveles de soltería que parecían pertenecer ya al pasado, porque en las nuevas generaciones adquieren otro significado: el del cambio.

\section{CUADRO 1}

Proporciones de solteros. Cataluña, 1975, 1981 y 1986

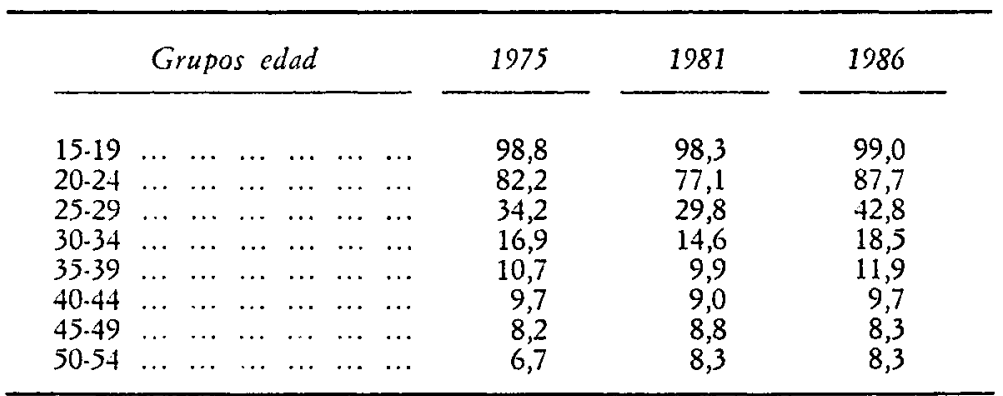

Las generaciones de varones nacidos entre 1921 y 1925 presentan, con solterías definitivas tan reducidas $-6,7$ por $100-$, los efectos favorables de su escaso número de efectivos al finalizar la guerra civil. Esta situación ya es irrepetible y los hombres que pertenecen a las generaciones posteriores se han casado en menor proporción. 
En el cuadro 2 figuran las proporciones de solteras de las mujeres que habitan en Cataluña, en las fechas padronales. Según los datos de 1981, el sexo femenino presentaba una menor proporción de solteras que el conjunto de toda España. La tendencia a un casamiento un poco más tardío de las catalanas se contrarrestaba con la elevada proporción de mujeres casadas muy jóvenes provenientes de otras comunidades de España. De esta manera, casi la mitad de las mujeres en Cataluña ya estaban casadas, si observamos las proporciones --según el estado civil- en el grupo de 20 a 24 años. En 1986, según los datos del padrón de habitantes, sólo una de cada tres estaba casada en ese grupo de edad. Esta postergación del matrimonio se manifiesta hasta los treinta y cinco años solamente. Más allá, las proporciones por estado civil resultan similares en las dos fechas y aún menores, como en el caso de los hombres, a partir de los cuarenta y cinco años. Evidentemente, el adelanto de los casamientos permitió a las jóvenes generaciones casarse antes de 1981. Las personas que tenían edades más elevadas y menores probabilidades de casarse no alteraron sus comportamientos, respecto a las tendencias observadas en la década anterior.

\section{CUADRO 2}

Proporciones de solteras. Cataluña, 1975, 1981 y 1986

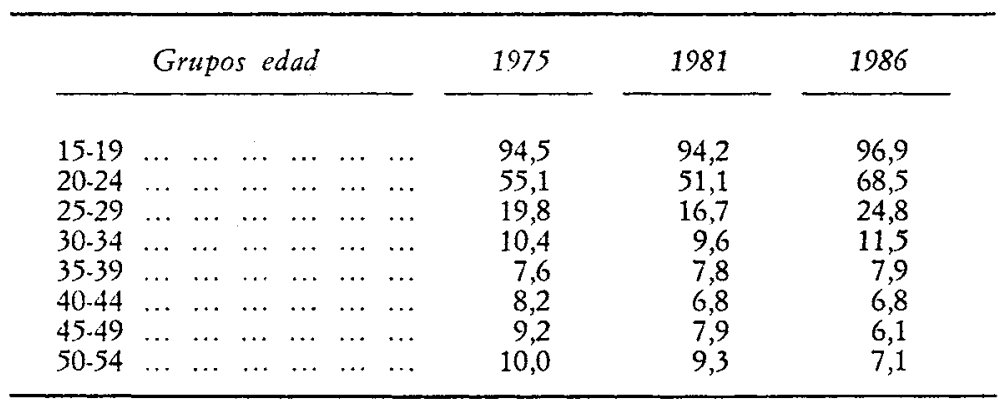

En los cuadros 3 y 4 aparecen las proporciones de solteros y solteras correspondientes a la provincia de Barcelona. Dado que por la importancia cuantitativa de esta población sus tendencias han marcado las del conjunto de la Comunidad Autónoma, estimo conveniente revisar y comparar sus indicadores respecto del total. Destacamos una conducta diferenciada por sexo. Tanto los hombres como las mujeres presentan una nupcialidad más tardía en la última fecha, pero existen diferencias destacables. Las proporciones de solteros en 1986 resultan superiores en Barcelona - provincia- respecto del conjunto de Cataluña en los dos primeros grupos de edad -15-19 y 20-24-, 


\section{CUADRO 3}

Proporciones de solteros. Barcelona (provincia), 1975, 1981 y 1986

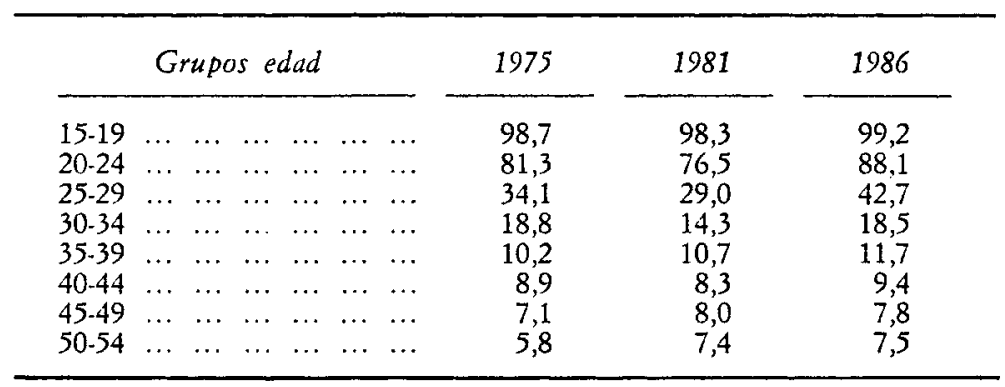

\section{CUADRO 4}

Proporciones de solteras. Barcelona (provincia), 1975, 1981 y 1.986

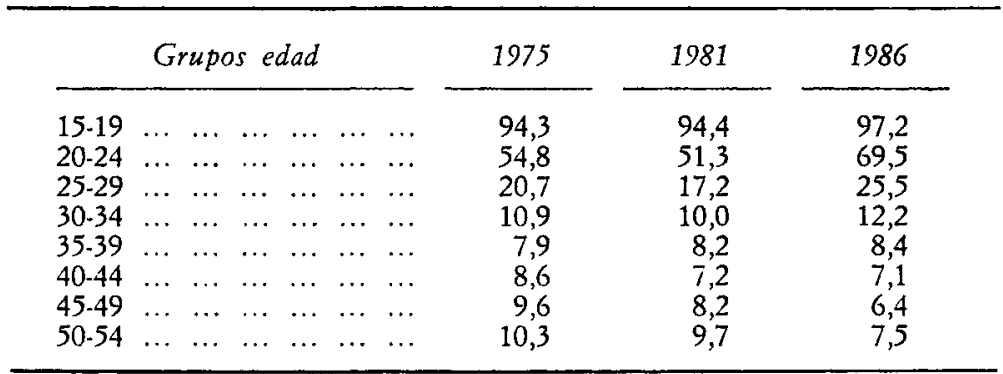

similares en los intermedios - 25-29 y 30-34 - e inferiores a partir de los treinta y cinco años. Los hombres no presentan un comportamiento homogéneo.

Es necesario destacar, sin embargo, la gran concentración de matrimonios de varones solteros en el segundo quinquenio de los años setenta ${ }^{2}$. El descenso observado en la década de los ochenta, en los distintos años calendarios, también proviene - además de la principal razón apuntada anteriormente de retraso en el matrimonio - de que una parte de los matrimonios que habrían de haberse celebrado en esta última década se adelantaron y

${ }^{2}$ En la provincia de Barcelona, la intensidad de la nupcialidad de solteros, medida transversalmente, fue, en el bienio $1975-76$, de 107 para los hombres y de 108 para las mujeres. En el conjunto de Cataluña, la intensidad fue superior a 100 , en ambos sexos, durante tres años, hasta 1977, inclusive, lo que apunta a una elevada concentración en esos años. 
tuvieron lugar anteriormente. El estado civil de los catalanes en 1986 confirma lo sostenido.

También hay que tener en cuenta que por efecto de la guerra civil los hombres tuvieron elevadas probabilidades de casarse y lo hicieron en una proporción irrepetible, por lo que la soltería definitiva - proporción de solteros a cincuenta años- de las nuevas generaciones tiende a elevarse, respecto de las otras y más antiguas generaciones. Los efectos de los cambios acaecidos en estos años se dejan sentir, sobre todo, en los jóvenes, que son los motores de estas transformaciones. En los más viejos juegan otros factores más importantes y determinantes, como el efecto generación.

Las proporciones de solteras en 1986, en la provincia de Barcelona, resultan similares a las catalanas en las mismas fechas. Las tendencias al rejuvenecimiento en el último quinquenio de los años setenta, y la contraria al envejecimiento durante la primera parte de los ochenta, son coincidentes. Cabe destacar, sin embargo, que existe una plena uniformidad en todos los grupos de edad: en todos los casos las mujeres de Barcelona han permanecido solteras en mayor proporción que en el total de Cataluña.

La mayor proporción de población urbana y el peso significativo de la capital, que incide negativamente en las probabilidades de contraer matrimonio de las mujeres, podrían ser la explicación de esta ligera diferencia. Hemos visto que no ocurre lo mismo con los hombres. Mientras que el sexo masculino presenta diferencias según la edad y la pertenencia a las distintas generaciones, en las mujeres se observan las mismas relaciones de mayor o menor en cualquier grupo de edad.

La disminución creciente de las proporciones de solteras en la provincia de Barcelona, según los datos del Padrón de 1975 y 1981, a las mismas edades, deja bien claro que la tendencia anterior a la presente década era de una mayor intensidad en la nupcialidad y de una menor edad media, frente a las reducidas posibilidades que tuvieron las generaciones afectadas por la guerra civil española - mujeres nacidas antes de 1935-.

En los cuadros 5 y 6 , de la ciudad de Barcelona, las proporciones de solteros y solteras son más elevadas que en la provincia y en el total de la Comunidad Autónoma. Las diferencias son cuantitativamente importantes a todas las edades y este retraso se traduce en una soltería definitiva superior. En el caso de los hombres, esta vez, la uniformidad es total y las proporciones superiores en todos los grupos de edad. Debemos destacar que en 1981 las generaciones en torno a la edad límite de la soltería - nacidas antes de la guerra civil, entre 1927 y 1936 - presentan porcentajes crecientes, respecto a 1975. Por lo anteriormente sostenido, es de esperar que en 1986 la soltería sea mayor a todas las edades. Sin embargo, las mujeres de más de cuarenta años -nacidas antes de 1946- muestran tendencias inversas respecto al conjunto, puesto que se observa una menor proporción de solteras en los tres últimos grupos de edad. 


\section{CUADRO 5}

Proporciones de solteros. Barcelona (ciudad), 1975, 1981 y 1986

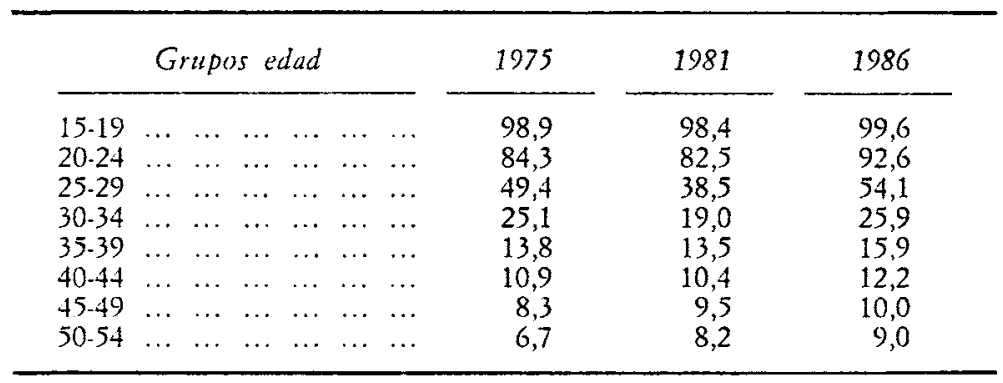

\section{CUADRO 6}

Proporciones de solteras. Barcelona (ciudad), 1975, 1981

$$
\text { y } 1986
$$

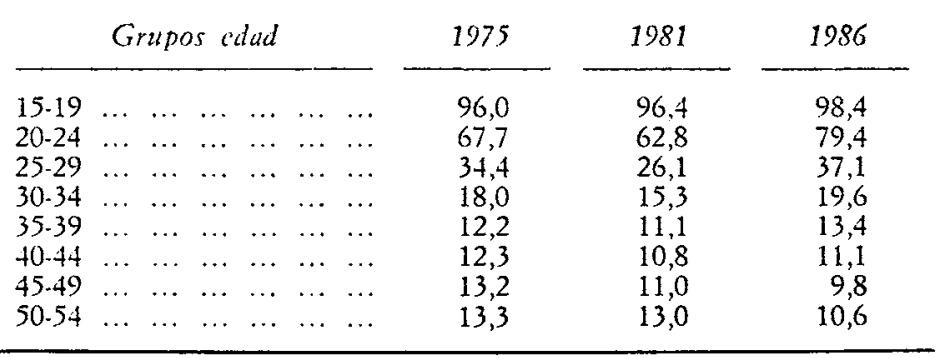

Las mujeres de la ciudad de Barcelona, al igual que los hombres, destacan por sus diferencias a las edades de casamiento más frecuentes. Que una de cada tres mujeres de Barcelona entre 25-29 años permanezca soltera frente a una de cada cuatro cata'anas apunta hacia actitudes y/o comportamientos relativamente dispares entre los habitantes de la capital y del conjunto de la Comunidad Autónoma.

Además, las proporciones se elevan en el último grupo de edad en 1986, tanto como en los años padronales anteriores. Este hecho ya no puede deberse al efecto generación - mayor soltería por efecto de la guerra civil- Dada la pérdida de población que experimenta la ciudad en estos años, debido a saldos migratorios negativos. El incremento de las proporciones podría deberse a una migración diferencial de mujeres casadas.

En otro trabajo anterior sobre la fecundidad (Sarrible, 1987) constaté 
la pérdida de efectivos de mujeres a partir de los cuarenta años y la ganancia en las primeras edades. El concepto de saldo migratorio uniformiza un flujo que no es idéntico por edad ni por estado civil, lo que tiene como consecuencia una transformación de la estructura de la población y no una simple diferencia de efectivos en el total.

En conclusión, si las generaciones se casaban con mayor frecuencia y más precozmente durante los años setenta siguiendo tendencias anteriores, a partir de la década de los ochenta estos comportamientos no tienen continuidad. Los jóvenes, sobre todo, han decidido retrasar el matrimonio. La intensidad final de la nupcialidad - medida en el método de Hajnal por la proporción de solteros/as a cincuenta años - todavía no se ha visto mayormente afectada por estos cambios. Por una parte, la elevada intensidad alcanzada durante los setenta influyó para que estas generaciones no presentaran elevadas proporciones a esas edades. Por la otra, el cambio se deja sentir, sobre todo, en los más jóvenes, y las personas mayores puede que continúen comportándose como lo hacían las generaciones de más edad.

\section{Las tasas de nupcialidad en los años calendarios}

Para completar el análisis de las proporciones que nos sugieren el estado de la población en fechas sucesivas se analizará a continuación la distribución por grupo de edad y sexo de los matrimonios de solteros y solteras en la década de los ochenta. Además de las tasas específicas he calculado las intensidades correspondientes a los distintos años - donde existen datos publicados- y las edades medias de cada sexo al contraer matrimonio.

En general, en los cuadros 7 y 8 observamos una disminución de las tasas

\section{CUADRO 7}

Tasas de nupcialidad de solteros. Cataluña, 1980, 1981, 1982, 1983,1985 y 1986

\begin{tabular}{|c|c|c|c|c|c|c|c|c|}
\hline \multirow{2}{*}{\multicolumn{3}{|c|}{ Grupos cdad }} & \multicolumn{6}{|c|}{ TASAS } \\
\hline & & & 1980 & 1981 & 1982 & 1983 & 1985 & 1986 \\
\hline $15-19$ & $\ldots \quad \ldots$ & $\ldots$ & 6,04 & 4,88 & 4,27 & 3,65 & 2,30 & 1,98 \\
\hline $20-24$ & $\ldots \quad \ldots$ & $\ldots$ & 73,57 & 65,79 & 59,93 & 50,54 & 41,92 & 38,84 \\
\hline 25.29 & $\ldots$ & $\ldots$ & 44,83 & 40,01 & 39,86 & 39,66 & 56,08 & 60,02 \\
\hline $30-34 \ldots$ & $\ldots \quad \ldots$ & $\ldots$ & 9,63 & 8,74 & 8,59 & 9,04 & 13,71 & 14,55 \\
\hline $35-39$ & $\ldots$ & $\ldots$ & 2,73 & 2,43 & 2,31 & 2,75 & 3,87 & 3,74 \\
\hline $40-44 \quad \ldots$ & $\ldots$ & $\ldots$ & 1,33 & 1,34 & 1,13 & 1,24 & 1,49 & 1,57 \\
\hline $45.49 \quad \ldots$ & $\ldots \quad \ldots$ & $\ldots$ & 0,67 & 0,72 & 0,72 & 0,75 & 0,93 & 0,88 \\
\hline $50.54 \ldots$ & $\ldots$ & $\ldots$ & 0,41 & 0,46 & 0,44 & 0,49 & 0,65 & 0,57 \\
\hline $55.59 \ldots$ & $\begin{array}{ll}\ldots & \ldots\end{array}$ & $\ldots$ & 0,35 & 0,48 & 0,42 & 0,31 & 0,40 & 0,29 \\
\hline 60 y más & $\ldots \quad \ldots$ & $\ldots$ & 0,77 & 1,39 & 1,69 & 0,88 & 0,71 & 0,63 \\
\hline
\end{tabular}




\section{CUADRO 8}

Tasas de nupcialidad de solteras. Cataluña, 1980, 1981, 1982, 1983,1985 y 1986

\begin{tabular}{|c|c|c|c|c|c|c|c|c|}
\hline \multirow{2}{*}{\multicolumn{3}{|c|}{ Grupos calad }} & \multicolumn{6}{|c|}{ TASAS } \\
\hline & & & 1980 & 1981 & 1982 & 1983 & 1985 & 1986 \\
\hline $15.19 \ldots$. & $\ldots \quad \ldots$ & $\ldots \quad \ldots$ & 26,73 & 22,09 & 19,17 & 16,90 & 11,69 & 10,53 \\
\hline $20-24 \ldots$ & $\begin{array}{ll}\cdots & \ldots \\
\ldots & \ldots\end{array}$ & $\begin{array}{ll}\ldots & \ldots \\
\ldots & \ldots\end{array}$ & 80,03 & 72,69 & 69,14 & 62,84 & 67,39 & 65,61 \\
\hline $25-29 \ldots$ & & $\begin{array}{ll}. . & \ldots\end{array}$ & 20,84 & 18,86 & 18,65 & 20,23 & 32,14 & 35,71 \\
\hline $30-34 \ldots$ & $\begin{array}{ll}\cdots & \cdots \\
\ldots & \ldots\end{array}$ & $\begin{array}{ll}\cdots & \cdots \\
\cdots & \cdots\end{array}$ & 4,70 & 3,92 & 4,27 & 4,36 & 6,70 & 6,77 \\
\hline $35-39 \ldots$ & $\ldots \quad \ldots$ & $\ldots \quad \ldots$ & 1,65 & 1,70 & 1,79 & 1,81 & 2,84 & 2,38 \\
\hline $40.44 \ldots$ & $\begin{array}{ll}\ldots & \ldots \\
\ldots\end{array}$ & .. $\quad \ldots$ & 0,83 & 0,79 & 0,78 & 0,91 & 1,27 & 1,17 \\
\hline $45.49 \ldots$. & $\ldots \ldots$ & $\ldots \quad \ldots$ & 0,65 & 0,68 & 0,65 & 0,62 & 0,77 & 0,58 \\
\hline $50.54 \ldots \ldots$ & & $\begin{array}{ll}\ldots & \ldots \\
\ldots\end{array}$ & 0,53 & 0,51 & 0,46 & 0,49 & 0,71 & 0,52 \\
\hline $55.59 \ldots$. & $\ldots \quad \ldots$ & ... $\ldots$ & 0,47 & 0,45 & 0,65 & 0,55 & 0,55 & 0,49 \\
\hline 60 y más. & $\begin{array}{ll}\ldots & \ldots \\
\ldots & \ldots\end{array}$ & $\begin{array}{ll}\cdots & \ldots\end{array}$ & 0,73 & 1,01 & 1,28 & 1,04 & 0,97 & 0,95 \\
\hline
\end{tabular}

en las primeras edades y un ligero incremento en las superiores. Dado que la nupcialidad de solteros en estos grupos es un fenómeno minoritario y casi residual, los aumentos en estos grupos no compensan, en ningún caso, los descensos de los más jóvenes.

Para el sexo masculino, el hecho más destacable es el cambio del grupo de edad modal al matrimonio a partir de 1985. Si los catalanes se casaban más frecuentemente entre veinte y veinticuatro años, hasta 1982, en el segun-

\section{CUADRO 9}

Tasas de nupcialidad de solteros.

Barcelona, 1980, 1981, 1982 y 1983

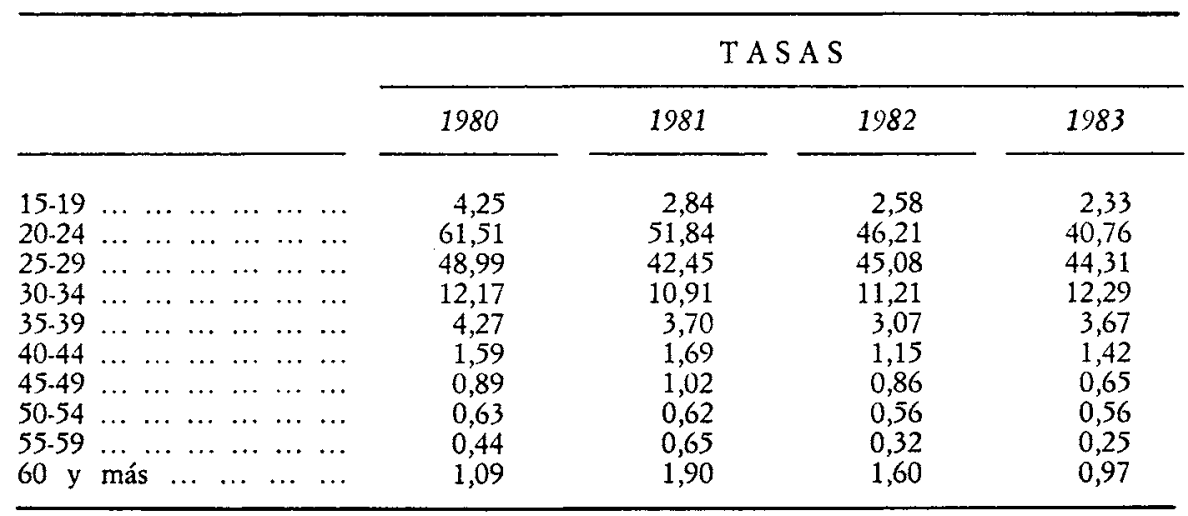




\section{CUADRO 10}

Tasas de nupcialidad de solteras. Barcelona, 1980, 1981, 1982 y 1983

\begin{tabular}{|c|c|c|c|c|}
\hline & \multicolumn{4}{|c|}{ TASAS } \\
\hline & 1980 & 1981 & 1982 & 1983 \\
\hline $15-19 \ldots \ldots \ldots \ldots$ & 18,63 & 14,52 & 11,69 & 10,99 \\
\hline 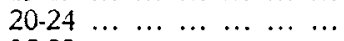 & 71,85 & 62,46 & 59,69 & 56,03 \\
\hline $\begin{array}{lllllll}25-29 & \ldots & \ldots & \ldots & \ldots & \ldots & \ldots\end{array}$ & 26,98 & 22,81 & 24,83 & 26,00 \\
\hline 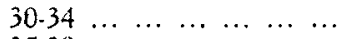 & 6,27 & 4,96 & 5,42 & 5,69 \\
\hline $\begin{array}{llllllll}35.39 & \ldots & \ldots & \ldots & \ldots & \ldots & \ldots\end{array}$ & 2,01 & 2,14 & 1,81 & 2,49 \\
\hline 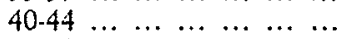 & 1,03 & 1,05 & 0,72 & 0,93 \\
\hline $\begin{array}{lllllll}45.49 & \ldots & \ldots & \ldots & \ldots & \ldots & \ldots\end{array}$ & 0,81 & 1,07 & 0,67 & 0,64 \\
\hline $\begin{array}{lllllll}50-54 & \ldots & \ldots & \ldots & \ldots & \ldots & \ldots\end{array}$ & 0,87 & 0,60 & 0,47 & 0,42 \\
\hline $\begin{array}{llllllll}55.59 & \ldots & \ldots & \ldots & \ldots & \ldots & \ldots\end{array}$ & 0,65 & 0,46 & 0,49 & 0,38 \\
\hline 60 y más $\ldots \ldots \ldots$ & 0,92 & 1,28 & 0,96 & 0,91 \\
\hline
\end{tabular}

do quinquenio lo hacen en el grupo de edad siguiente. El descenso de la intensidad de la nupcialidad ha estado motivado por una postergación y no por un rechazo al matrimonio. Los hombres que han pospuesto la unión han comenzado a casarse. El incremento de las tasas de nupcialidad a partir de los treinta años constituye un claro ejemplo de ello, hecho que se observa ya en 1983.

Es cierto que en términos de intensidad todavía estamos muy lejos de las registradas en la década del setenta. Pero hay que recordar que éstas fueron excepcionales y que posiblemente no vuelvan a repetirse. La caída de la intensidad se ha detenido, pero el incremento de las edades medias no. La recuperación ha comenzado. Como las generaciones que han postergado el casamiento tienen más edad, es lógico que ésta continúe incrementándose, al menos por un tiempo.

Las tasas de nupcialidad de solteras en Cataluña muestran las mismas tendencias que las correspondientes al otro sexo: descenso generalizado hasta 1982; ligera recuperación en los grupos de edad intermedia en 1983; a partir de 1985, reducción hasta veinticinco años y posterior incremento. En el caso de las mujeres no se observa una transformación tan radical como el cambio de la edad modal; aparentemente, está muy lejos de ello. La postergación de los matrimonios continúa en las jóvenes generaciones, mientras que las no-tan-jóvenes se están casando con una frecuencia dos veces superior, grosso modo, a las tasas observadas en los cuatro primeros años de la década - de 25 a 44 años-.

La intensidad y el calendario de hombres y mujeres -cuadro 11 y gráficos I y II - siguen las mismas tendencias: descenso de la intensidad, calcu- 


\section{CUADRO 11}

Nupcialidad: intensidad y calendario.

Matrimonios reducidos y edad media.

Cataluña, 1980, 1981, 1982, 1983, 1985 y 1986

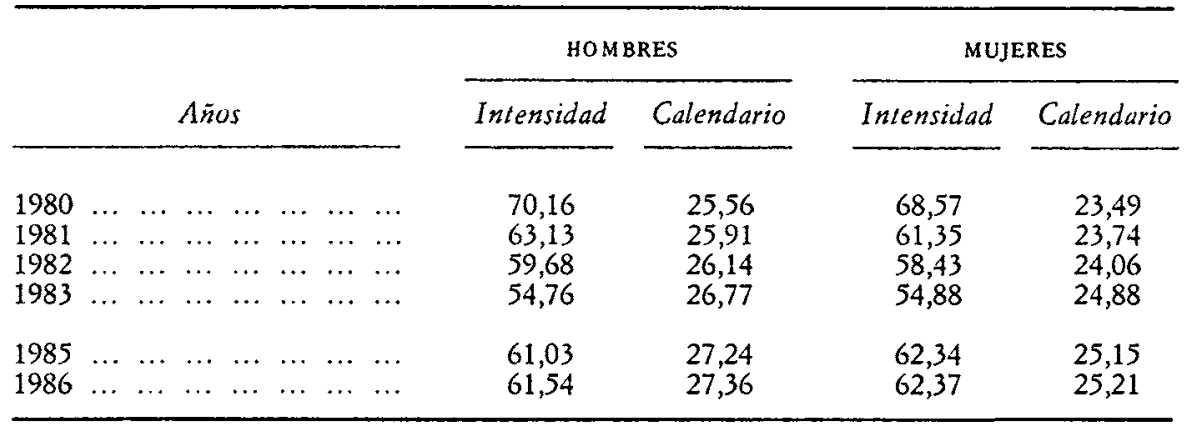

\section{GRAFICO I}

Nupcialidad en Cataluña en los ochenta (Intensidad por sexo)

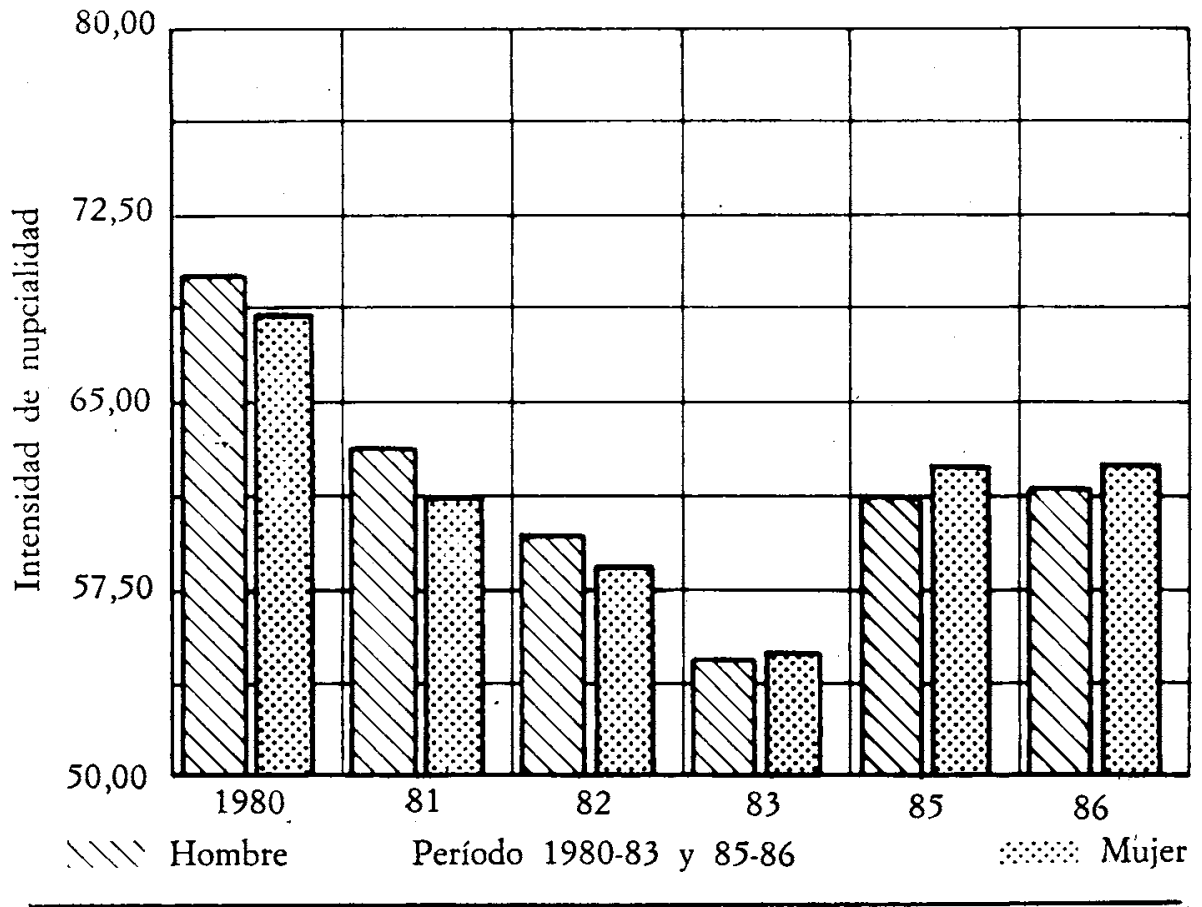


Los datos sobre la ciudad de Barcelona sólo llegan hasta 1983. Sin embargo, estos años parecen de transición. En 1982 ya se observa una ligera recuperación en las mujeres de veinticinco a treinta y cuatro años, mientras continúa el descenso en los otros grupos. Esta tendencia se generaliza a otros grupos de edad intermedios y a los dos sexos en el año siguiente. En el conjunto de Cataluña la tendencia es similar, salvo que la recuperación femenina se observa entre los 30-39 años, en 1982.

Las tendencias generales en Barcelona son las mismas que en Cataluña: descenso de la intensidad, sobre todo en las jóvenes generaciones. En los cuadros 9 y 10 observamos que en el año 1982 ya se produce un incremento en los grupos de edad 25.29 años, tanto en los hombres como en las mujeres. En el caso del sexo masculino, el cambio del grupo de edad modal se produce en 1983. En el caso de las mujeres constatamos las mismas tendencias; pero, al igual que en el conjunto de Cataluña, estamos todavía bastante lejos de un cambio en la edad modal. El incremento de las tasas de los dos sexos a partir de los veinticinco años y en los grupos de edad intermedios se generaliza en 1983, pero no llega a paliar el descenso (gráfico III).

\section{GRAFICO III}

Nupcialidad en Barcelona

(Intensidad por sexo)

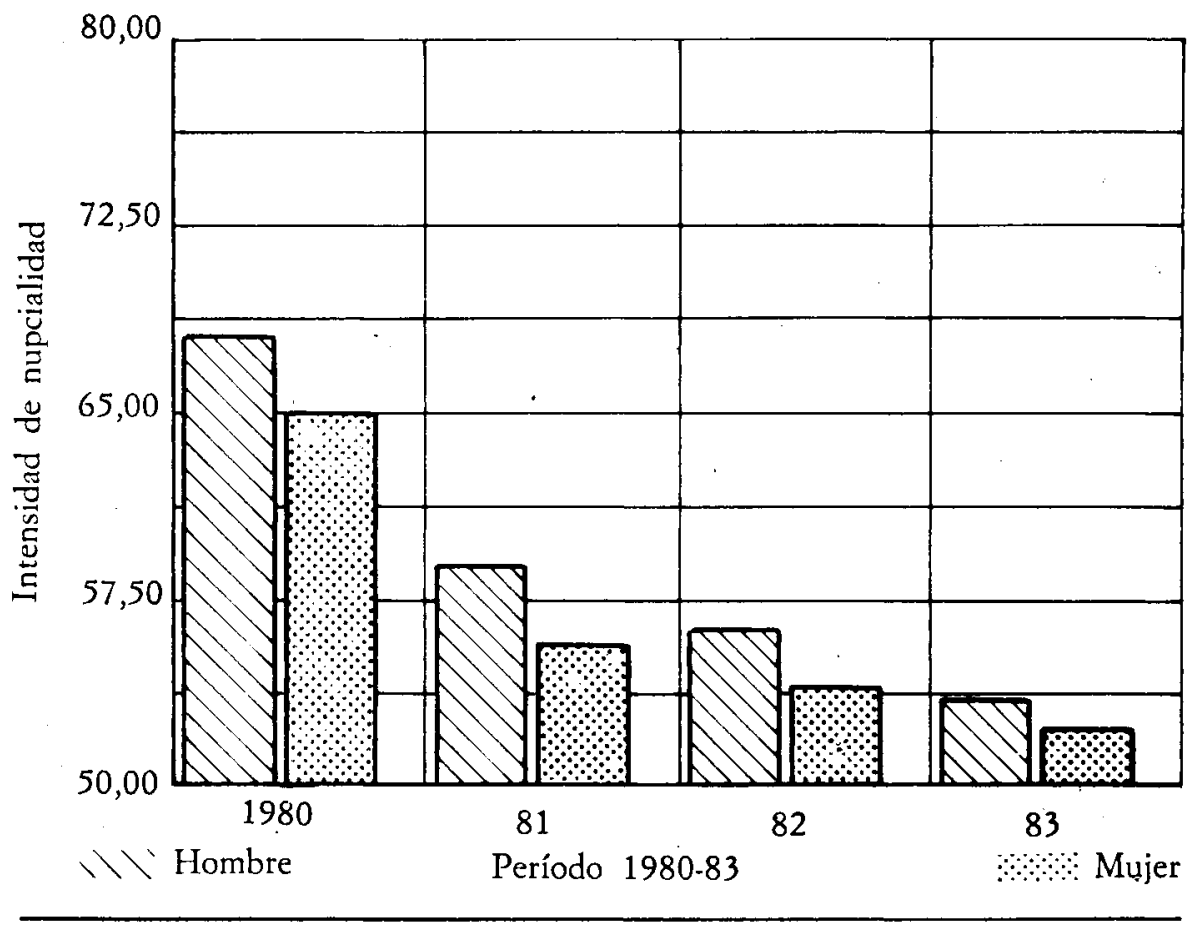


En conclusión, las tasas de nupcialidad de solteros permiten confirmar lo observado en las proporciones. Existe un descenso en los casamientos en la primera parte del período y un incremento en la edad media al matrimonio. La baja intensidad no representa un revulsivo, puesto que la recuperación ya ha comenzado. El retraso que arrastraban las jóvenes generaciones ha incrementado las edades medias de una manera muy rápida, ya que estas transformaciones, en general, se producen sólo en largos períodos de tiempo. El cambio en la edad modal para el sexo masculino resulta significativo, pero dada la precocidad con que se casan las mujeres y las diferencias entre los dos sexos, no considero factible, al menos en el corto plazo, que ocurra lo mismo.

\section{DISCUSION}

Durante el presente siglo, España se ha caracterizado por una edad tardía al matrimonio. El proceso de rejuvenecimiento fue, sin embargo, relativamente rápido; en todo caso, más que en la fecundidad. De una edad media de 28,8 para el sexo masculino y de 26,1 para el femenino en 1960 , se ha pasado, en veinte años, a 25,2 y 23 , respectivamente (Eurostat, 1986). Sin embargo, en la presente década asistimos al fenómeno inverso: los españoles permanecen solteros en mayor proporción, y si deciden casarse lo hacen más tarde.

En Cataluña estos cambios han ocurrido después de un período de una alta intensidad de la nupcialidad, por lo que tiene visos aparentes de mayor radicalidad. Sin embargo, hay que tener presente que los casamientos celebrados hacen salir de observación a los solteros y que la gran concentración del segundo quinquenio de los setenta tenía que provocar un descenso de la intensidad de los casamientos, a menos que la tendencia al rejuvenecimiento se prolongara indefinidamente - matemática pero no socialmente posible-

En la actualidad, e] descenso se ha detenido y la recuperación ha comenzado. Los matrimonios temporalmente postergados se están realizando, aunque no todos, como era de esperar. Los retrasos, tanto en nupcialidad como en fecundidad, siempre han ido aparejados con una menor intensidad final.

En la ciudad de Barcelona, donde la soltería es mayor, los indicadores transversales de intensidad son de menor magnitud que en Cataluña en los mismos años. Complementariamente, las edades medias resultan superiores que en el caso catalán.

Parte del descenso de la fecundidad española, catalana y barcelonesa puede explicarse por la disminución de parejas recién casadas. Si hay una recuperación de los matrimonios, pero no de la fecundidad, implica que los comportamientos también se han transformado en materia de reproducción y el intervalo protogenésico podría incrementarse.

Las nuevas generaciones no se comportan como las anteriores. La similitud que observábamos antes entre las próximas, puede haberse quebrado. Los jó- 
venes hoy no rechazan el matrimonio, pero llegan más maduros a él. Lo postergan como postergan la llegada de la descendencia. Tienen mayor libertad que las anteriores generaciones. Quizás sean más felices permaneciendo en casa de sus padres y por ello, postergan la partida.

También es cierto que la permanencia en el sistema educativo puede contribuir a alargar la estancia en la casa paterna. El mayor tiempo medio en que tardan en encontrar su primer empleo puede haber contribuido también a todas estas transformaciones temporales. Sin embargo, yo no consideraría a los dos últimos factores señalados como única razón de los cambios acaecidos. Posiblemente han coadyuvado, pero los comportamientos frente a una institución tan formal como el matrimonio no pueden depender exclusivamente de ellos.

No es aconsejable tanto alarmismo en cuanto al futuro de la reproducción de la población española. Ya se ha constatado una inflexión de las tendencias. Lo que no se puede esperar es que los comportamientos de las nuevas generaciones reproduzcan otros anteriores que corresponden a tiempos pasados. Estudios realizados sobre parejas casadas demasiado jóvenes inciden en la mayor inestabilidad y en probabilidades relativamente elevadas de divorciarse (Kiernan, 1986). Si los jóvenes de hoy se casan más tarde y reflexionan más antes de hacerlo, no puede significar, en ningún caso, que hayan elegido un modelo equivocado.

\section{A MODO DE CONCLUSION}

Los cambios en la nupcialidad han sido constantes. Si recurrimos al análisis transversal, las oscilaciones han sido significativas. Sin embargo, el cambio en las generaciones no se ha producido con igual rapidez e intensidad.

Los jóvenes de los ochenta han marcado una línea divisoria y sus comportamientos ya no se asemejan a los anteriores. Un retraso en contraer matrimonio y una elevación de la edad media han sido los cambios más significativos. Tanto los hombres como las mujeres han decidido permanecer solteros más tiempo. A las dificultades económicas, reales y que no puedo negar, se ha solapado un cambio de actitud. No hay tanta prisa por casarse como antes. La recuperación de los últimos años demuestra que no es posible hablar de rechazo, sino sólo de una postergación.

Si este proceso continúa, podremos esperar una ligera recuperación de la fecundidad transversal y un cambio más radical en las actitudes de las nuevas generaciones que estos años acaban de entrar en observación. Es necesario esperar varias décadas para confirmar o rechazar estas previsiones. 


\section{BIBLIOGRAFIA}

Díez Nicolós, Juan (1988): «La población española», Anuario El País 1988, Madrid, p. 407.

Capellades, Joaquim, y Olivares, Dolors (1986): «La nupcialidad a Catalunya, 1985», Estadistica $i$ Societat, full núms. 57-58, CIDC, Barcelona, nov.-dec.

Eurostat (1986): Demograpbic Statistics, Oficina Estadística de las Comunidades Europeas, Luxemburgo.

Goody, Jack (1985): L'évolution de la famille et du mariage en Europe, Armand Colin, París.

Hajnal, John (1953-54): «Age at marriage and Proportions Marrying», Population Studies, vol. 7, pp. 112-136.

Kiernan, Kathleen (1986): «Teenage Marriage and Marital Breakdown: A Longitudinal Study», Population Studies, Londres, 40, pp. 35-54.

SARRIBLE, Graciela (1987): Estudio de la fecundidad en la población nativa y migrante de la ciudad de Barcelona, Colección Micro, Universidad de Barcelona, Barcelona.

- (1988): "Changements dans la fécondité différencielle des femmes migrantes: une interpretation alternative - l'exemple de Barcelone», communication au colloque sur La transition démograpbique au pays de la méditerranéen, Université de Nice, mai.

Soler Serratosa, Joan (1987): «Los cambios de la familia en la sociedad urbana», Barcelona, 3, diciembre-marzo, Ayuntamiento de Barcelona, Barcelona, pp. 114-117. 